\title{
Following Camelia's life: la memoria di Camelia Matatia come percorso di alternanza scuola-lavoro
}

L'articolo tratta di un'attività di alternanza scuola-lavoro svolta dalla classe VB del Liceo Righi nel corso dell'anno scolastico 2017/18 e costruita attorno alla vicenda di Camelia Matatia e alla mostra a lei dedicata: Following Camelia's life. Si tratta di un percorso didattico che ha coinvolto gli studenti nell'allestimento e nella realizzazione di visite guidate della mostra, che rende conto dello stravolgimento della vita di una ragazza ebrea in seguito alla promulgazione delle leggi razziali del 1938. Dopo una descrizione della vicenda storica, degli obiettivi e della struttura della mostra, si passa a un resoconto dello svolgimento del progetto, con analisi dei punti di forza e debolezza dell'esperienza in vista di future riproposizioni.

The article is about a work-related learning project, carried out by class VB of Liceo Righi during the school year 2017/2018. It is built around Camelia Matatia and the show dedicated to her: Following Camelia's life. The activity consisted in a scholar project, which saw the students involved in setting up the exhibit and preparing guided tours to the show, which narrates of the overturning of a Jewish girl's life after the promulgation of racial laws in 1938. After a concise description of the historical setting, the objectives and the structure of the show, the article moves on to talk about the unfolding of the project, with an analysis of the main strengths and weaknesses of the experience, in view of new future proposals. 


\section{La vicenda storica: la vita di Camelia}

Il progetto didattico nasce nel 2015 grazie alla collaborazione tra Istituto storico Parri, Assemblea legislativa Emilia-Romagna, Anne Frank House di Amsterdam e liceo Galvani di Bologna. L'attività è basata sulla ricostruzione della vita di Camelia Matatia, nata nel 1926 a Forlì da una famiglia benestante che fu vittima delle leggi razziali e della deportazione. Il padre, Nissim Matatia, ebreo greco originario di Corfù, era emigrato in Italia insieme ai fratelli Leone ed Eliezer negli anni Venti. I tre avevano fatto rapidamente fortuna grazie all'acquisto di due pelliccerie a Forlì e Faenza. Completavano la famiglia di Camelia la madre Matilde Hakim, ebrea bolognese di origine turche, e i fratelli, rispettivamente maggiore e minore, Beniamino, detto Nino, e Roberto.

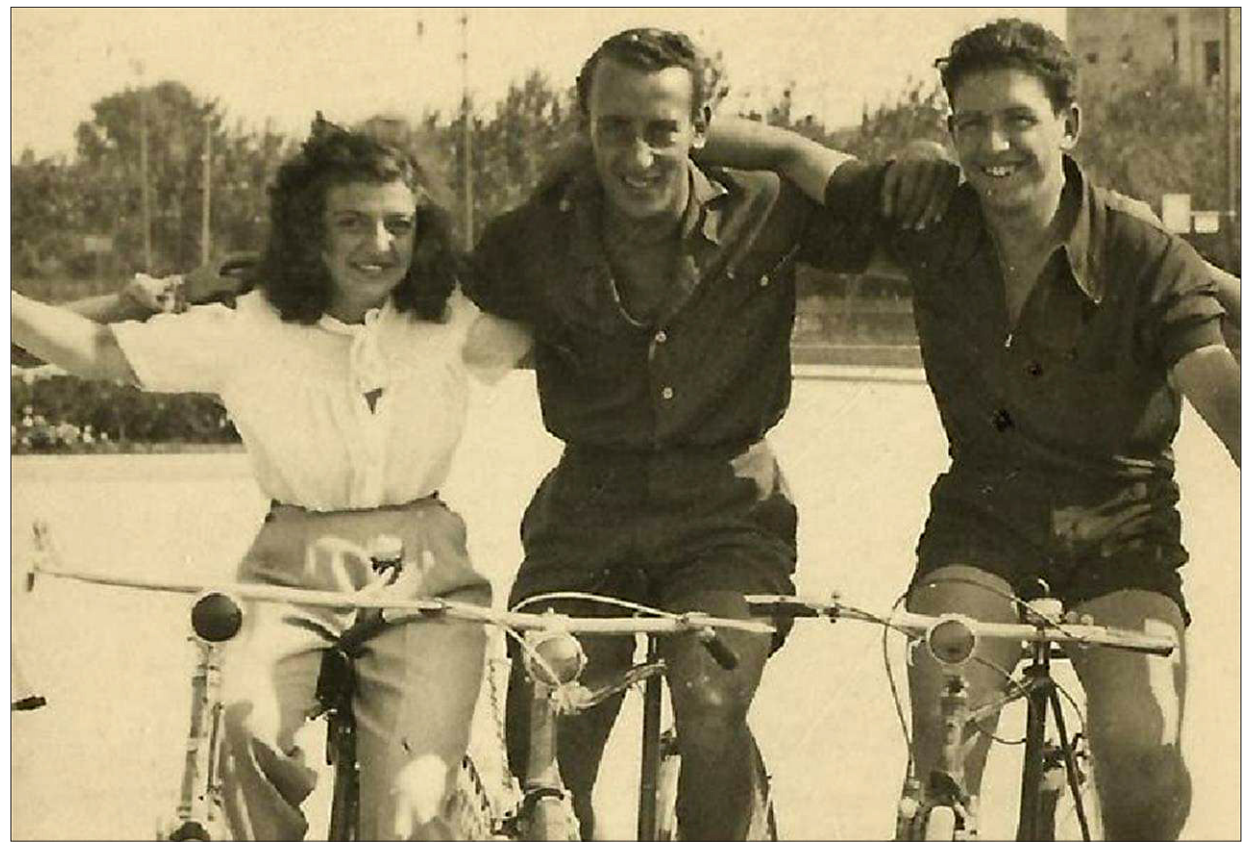

Camelia e Beniamino Matatia e un loro amico, anni Trenta.

I Matatia conducevano una vita serena e agiata, testimoniata dalla proprietà di una villa estiva a Riccione lungo viale Ceccarini, con giardino esteso fino alla spiaggia. Poco distante sorgeva Villa Margherita, che divenne negli anni Trenta la residenza estiva di Benito Mussolini. In agosto la presenza del Duce rendeva la cittadina un polo di mondanità, ricco di eventi e frequentato dalle alte cariche del regime e degli operatori dell'Istituto Luce. Per molto tempo la famiglia Matatia era pienamente inserita in quell'alta società: Nissim poteva vantare amicizie 
altolocate tra i gerarchi fascisti, le cui mogli erano clienti della sua pellicceria. Talvolta capitava che Camelia giocasse in spiaggia insieme a Romano Mussolini, il figlio del Duce.

La villa, simbolo del successo sociale dei Matatia, divenne tuttavia l'inizio della rovina della famiglia. Mentre il legame tra il Duce e il Führer andava rafforzandosi, Nissim iniziò a ricevere ripetute convocazioni presso la Questura di Bologna. Qui funzionari dell'Ovra gli intimarono, ricorrendo a minacce di ritorsioni contro la sua famiglia, di vendere la casa di Riccione, divenuta fonte d'imbarazzo per 1'Italia data la vicinanza a Villa Margherita: non era ammissibile che una famiglia di origini semite potesse abitare a pochi metri dal Duce.

Qualcosa era cambiato e gli ebrei erano improvvisamente in pericolo: le conoscenze altolocate di Nissim gli suggerirono di chiudere il negozio e abbandonare l'Italia insieme a tutta la famiglia. La pellicceria di Forlì, attraverso un boicottaggio, perse improvvisamente la clientela costringendo Nissim alla chiusura. Mentre i fratelli Leone ed Eliezer Matatia diedero ascolto agli avvertimenti, rifugiandosi, rispettivamente, in Svizzera e Francia, Nissim, orgoglioso e ingenuamente fiducioso delle proprie supposte amicizie, scelse di restare in Italia, tenendo testa alle minacce dell'Ovra e senza vendere la casa.

La dimostrazione di quanto il suo ottimismo fosse mal risposto arrivò nel settembre 1938 con la promulgazione delle leggi razziali: Camelia, Nino e Roberto furono costretti ad abbandonare la scuola pubblica, così la famiglia Matatia si trasferì nella casa di via Ugo Bassi a Bologna, dove la comunità ebraica si era organizzata per permettere ai giovani la prosecuzione degli studi.

La situazione dei Matatia peggiorò ulteriormente nel novembre del 1943 quando Nissim, unico membro della famiglia a non avere cittadinanza italiana, fu arrestato in seguito a un controllo ed espulso a Kerkyra (Corfù), sua città natale. Dalla Grecia, forzato dalle insistenti minacce dell'Ovra al suo commercialista, dovette infine arrendersi a vendere la villa di Riccione, ricavando per altro un prezzo risibile rispetto al valore di mercato. Intanto, per scampare allo stretto controllo della polizia fascista a Bologna, Matilde e i figli trovarono rifugio a Savigno.

Nel 1940, incapace di restare lontano da Matilde e i bambini, Nissim rientrò clandestinamente in Italia. La comunità ebraica gli fornì un rifugio, da cui usciva periodicamente per vedere la moglie e i figli, in incontri a due in luoghi pubblici per non destare sospetti. Nel novembre del 1943, proprio durante uno di questi incontri, Nissim e Roberto vennero arrestati. Trascorsero un periodo nel carcere di Verona fino al 6 dicembre 1943 e furono entrambi deportati ad Auschwitz. Lo stesso destino colpì il resto della famiglia: 1'arresto di Camelia, Matilde e Roberto avvenne il $1^{\circ}$ dicembre 1943 a Savigno, in seguito alla delazione di una contadina 
del luogo. La madre e i figli furono prigionieri nei penitenziari di Bologna, Forlì, Ravenna e Milano, da cui vennero infine fatti salire sul treno per Auschwitz dal tristemente noto Binario 21.

Nissim morì il 27 aprile 1944, Roberto il 18 gennaio 1945. Nino fu l'unico Matatia a sopravvivere, ma morì poco dopo il rientro in Italia a causa delle pessime condizioni di salute causate da deportazione e internamento. Delle vite di Camelia e Matilde non sono rimaste tracce successive all'immatricolazione ad Auschwitz, due inequivocabili vuoti tra i milioni di esistenze annientate tra quelle mura.

\section{I documenti per la ricostruzione storica}

Nel momento di massima difficoltà della sua famiglia, Camelia trovò l'unica consolazione dall'incontrò con Mario, un giovane di Zola Predosa conosciuto sulla corriera che li portava a scuola. Tra i due nacque una relazione sentimentale testimoniata dalle numerose lettere che Camelia scrisse al ragazzo. Fu proprio Mario, durante la vecchiaia, a far visita a Roberto Matatia, imprenditore faentino discendente di Camelia, nel suo negozio di Faenza, dove gli consegnò una carpetta gialla che custodiva le lettere del suo amore giovanile.

Negli scritti è testimoniato il disagio di Camelia nel subire la persecuzione razziale per colpe inesi-

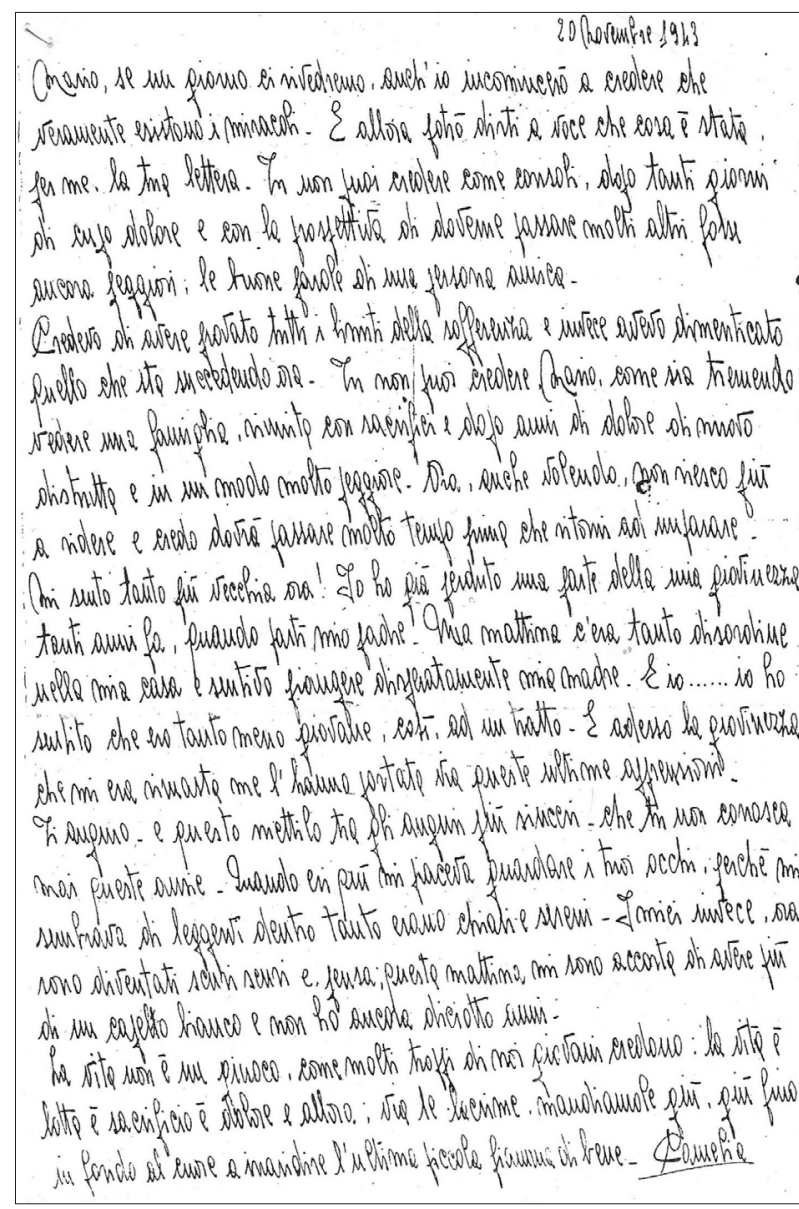

Una delle lettere di Camelia (per gentile concessione di Roberto Matatia). 
stenti, «un marchio disgraziato che nemmeno la scolorina del tempo potrà cancellare». Il testo esprime la grande maturità della ragazza, che, dati l'esilio di Nissim, il crollo emotivo di Matilde e di Nino e la tenera età di Roberto, fu forzata a diventare la figura di riferimento della famiglia. Le parole che ha lasciato mostrano la trasformazione di una bambina in una donna disincantata, derubata della propria giovinezza e rassegnata di fronte a un ingiusto e tragico destino.

E proprio queste lettere hanno rappresentato il motore della ricerca di Roberto Matatia. La raccolta di documenti e di testimonianze e sulla propria famiglia ha permesso la realizzazione del libro I vicini scomodi (uscito nel 2014, per la casa editrice Giuntina), nel quale Roberto cerca di ricostruire la vita di Camelia. La pubblicazione riscuote un inatteso successo e riceve interesse da più parti, ciò che induce l'autore a dedicarsi attivamente alla testimonianza della vicenda di Camelia che continua a portare anche oggi nelle scuole di tutt'Italia insieme a sua moglie.

\section{Presentazione del progetto: storia e struttura della mostra}

Following Camelia's life è la mostra dedicata alla vita di Camelia Matatia sviluppata e gestita da studenti in tutte le sue fasi: allestimento, promozione e guida delle visite. Nata nel 2015 per opera di Istituto storico Parri, Assemblea E-R e Anne Frank House di Amsterdam, la mostra è stata pensata e sviluppata da alcuni studenti del Galvani, in seguito a un percorso didattico sulla Shoah presso la Anne Frank House di Amsterdam e alla lettura de I vicini scomodi. Following Camelia's life è stata inaugurata presso il Liceo Galvani poi ospitata in Sala Borsa. L'anno successivo è stata allestita nella biblioteca del liceo Augusto Righi di Bologna, dal 12 febbraio al 20 maggio 2018.

La mostra si compone di 12 pannelli a sviluppo verticale divisi in due sezioni: nella parte inferiore è riportata la ricostruzione storica, corredata da foto e documenti, degli eventi della vita della famiglia Matatia; nella parte superiore sono presenti foto di giovani volti, il primo di Camelia e i successivi degli studenti del Galvani. Sotto ogni viso è trascritto a mano un estratto delle lettere di Camelia inviate a Mario.

Il tour della mostra ha una durata media di mezz'ora e si divide in tre momenti principali:

- Introduzione (3 minuti): gli studenti, nel ruolo di guide, accolgono il pubblico, si presentano e spiegano brevemente com'è nata la mostra, come si struttura e come si svilupperà la visita. 

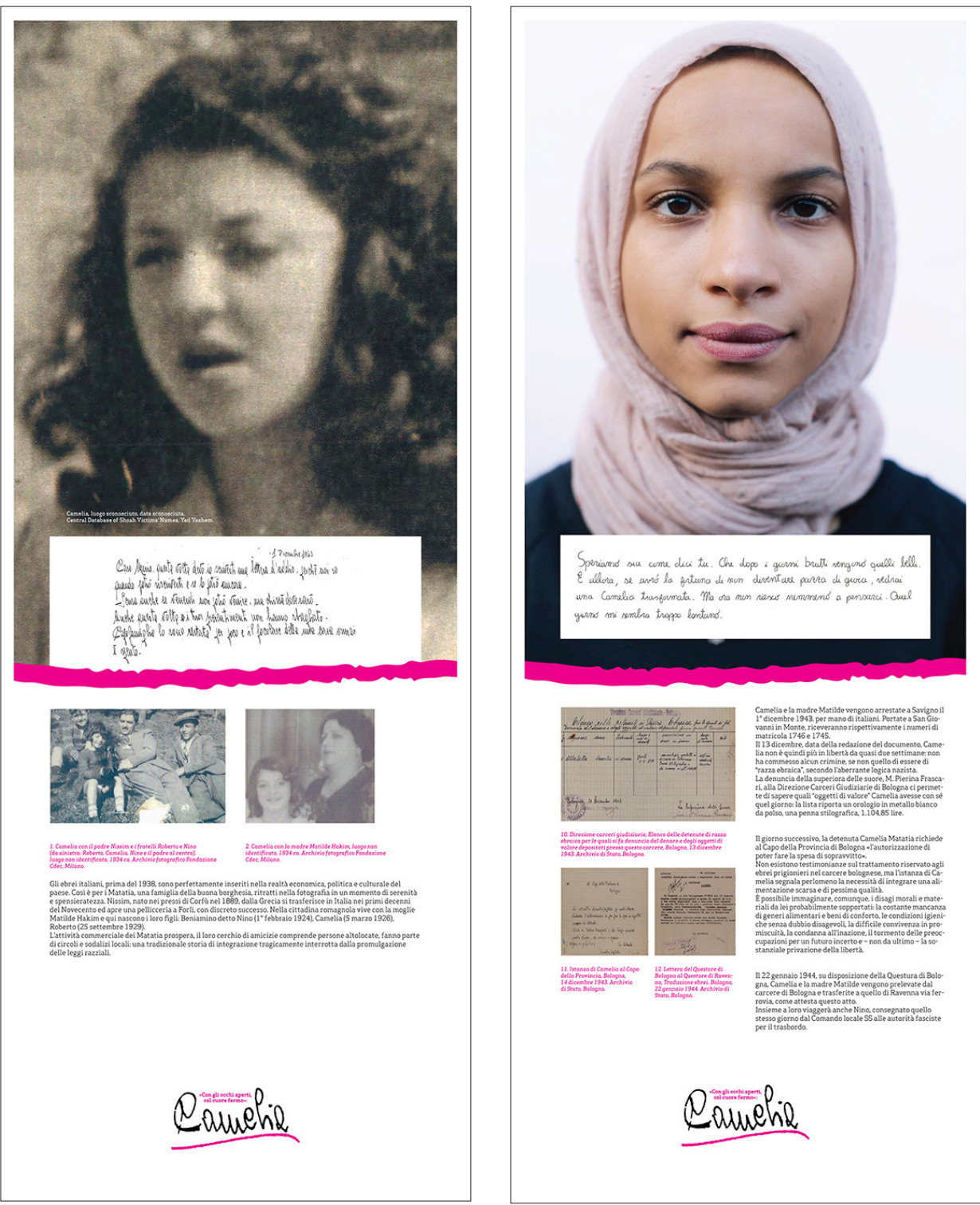

- Visita (20-25 minuti): gli studenti accompagnano il pubblico in un percorso attraverso i pannelli mentre espongono le tappe della vita di Camelia. Alla metà e in conclusione della spiegazione $\mathrm{i}$ visitatori hanno alcuni minuti per permettere un'esplorazione in autonomia.

- Riflessioni finali (5 minuti): gli studenti radunano il pubblico per trarre le conclusioni del lavoro svolto: raccontano del legame di vicinanza empatica costruito con Camelia, simboleggiato dalle foto dei giovani volti unite agli estratti delle lettere, e dell'impegno da loro intrapreso come testimoni della memoria della ragazza e della sua famiglia. 
L'edizione della mostra del liceo Righi ha inoltre visto un'aggiunta alla fase conclusiva: attraverso un metaforico passaggio di staffetta, i visitatori sono invitati dagli studenti a proseguire l'opera di testimonianza, lasciando per iscritto su alcuni post-it colorati i propri commenti e pensieri suscitati dalla mostra. Questi foglietti sono raccolti su un cartellone, che continuerà a essere parte della mostra nelle future riproposizioni, a segno della pluralità dell'impegno a tenere viva la testimonianza di Camelia.

\section{Obiettivi per gli studenti e per il pubblico}

In conformità con le linee guida dalla legge 107 del luglio 2015 dell'alternanza scuola-lavoro, il progetto intende proiettare gli studenti in un ambiente professionale simulato, nel quale sviluppare competenze trasversali di autonomia organizzativa, creatività e collaborazione tra compagni. Alla tradizionale didattica trasmissiva dell'aula, che prevede la sola lezione seguita dallo studio a casa, lo studente ha così la possibilità di alternare il contatto con un ambiente professionale poco esplorato, che richiede responsabilizzazione e diretta interazione in una situazione dinamica entro un gruppo di lavoro. Come obiettivi specifici, l'attività incentrata su Following Camelia's Life si focalizza sullo sviluppo delle seguenti aree di apprendimento:

- Capacità di organizzazione e gestione di un evento culturale: gli studenti si approcciano a questo settore professionale diventando protagonisti attivi di tutte le fasi logistiche di preparazione della mostra, che allestiscono e pubblicizzano attraverso lo sviluppo di materiale cartaceo e multimediale.

- Capacità di esposizione al pubblico: gli studenti rielaborano i dati di una vicenda storica ricostruita attraverso fonti letterarie e reperti memoriali e li trasmettono al pubblico, divenendo interpreti consapevoli di un processo di testimonianza.

- Sensibilizzazione sul tema delle leggi razziali: quest'obiettivo è da considerare in condivisione tra studenti e pubblico. Following Camelia's life intende trasmettere, attraverso la narrazione della vita di una giovane vittima della Shoah, l'impatto che le leggi razziali ebbero sulla vita degli ebrei italiani e del meccanismo attraverso cui permisero l'espulsione dalla società di coloro che ne erano membri radicati. Tutto ciò in un clima d'indifferenza, se non di complicità attiva, di buona parte della popolazione. La memoria della vicenda di Camelia diventa quindi un pretesto, non per ribadire l'annoso e fallace "imparare la storia per non ripetere gli stessi gli errori”, ma per comprendere, attraverso lo sguardo innocente di una vittima, la velenosa diffusione di una dinamica di razzismo nella società, nei reali e quotidiani atti delle persone che voltarono le spalle e si fecero carnefici dei 
loro vicini, divenuti improvvisamente scomodi. Nel caso degli studenti, l'attività rappresenta inoltre un'occasione di comprendere a un livello più profondo questa delicata parte del programma curricolare di storia.

\section{L'esperienza con il liceo Righi}

Nel corso dell'anno scolastico 2017-2018, Following Camelia's life è stata proposta e svolta come attività di alternanza scuola-lavoro della classe VB del liceo Augusto Righi di Bologna. Hanno coordinato il progetto due tutor, rispettivamente esterna e interna, Agnese Portincasa e Marianna Tubi. Lo svolgimento del percorso didattico ha coperto 30 ore di alternanza scuola-lavoro, il 33\% delle 90 ore curriculari previste per il triennio dei licei.

\section{Preparazione}

La preparazione della mostra si è svolta nel corso della terza settimana di gennaio 2018. Dopo essersi approcciata alla vicenda storica attraverso la lettura de I vicini scomodi, la classe ha partecipato a un incontro di formazione presso l'Istituto storico Parri, dove il progetto è stato presentato e sono state fornite le istruzioni relative alle sue modalità di sviluppo. Su gentile concessione di Roberto Matatia, gli studenti hanno inoltre potuto accedere alle lettere di Camelia, la cui lettura ha rappresentato un passaggio fondamentale per rafforzare nella classe la comprensione dell'obiettivo della mostra. Riconoscendo se stessi nella scrittura spontanea di una coetanea, sofferente per la privazione del proprio contesto adolescenziale costituito di familiari, amicizie e amori, gli studenti trovano un punto di contatto, pur indiretto, con il soggetto della mostra che andranno a presentare. L'avvicinamento empatico tra narratore e narrato diventa quindi la chiave fondamentale per farsi protagonisti consapevoli del processo di testimonianza e trasmetterlo al pubblico.

L'organizzazione del lavoro ha previsto la divisione degli studenti in 4 gruppi addetti, rispettivamente, al trasporto, montaggio e smontaggio, allestimento; visite guidate; progettazione; gestione e realizzazione delle azioni. La compartimentazione degli incarichi in fase di preparazione non ha comunque precluso la collaborazione tra gruppi, sollecitati, anzi, all'aiuto reciproco. I tutor hanno seguito e orientato l'impostazione del compito di ciascun gruppo di lavoro, lasciando successivamente agli studenti lo spazio per lavorare in autonomia. La classe ha avviato i lavori trasferendo il materiale della mostra dall'Istituto Storico Parri alla 
biblioteca del liceo Righi, dove gli studenti allestitori hanno montato la mostra con il supporto degli operatori del Parri. Questo gruppo di lavoro, per evidenti ragioni tecniche legate al ruolo, ha ricevuto maggior supervisione. Per quanto riguarda l'ambito della promozione, un gruppo ha realizzato e distribuito per le strade i volantini dell'evento, mentre un altro ha creato il video promozionale, le pagine Facebook e Instagram e un indirizzo email di riferimento per le prenotazioni delle visite. Le guide si sono infine divise i momenti della presentazione, hanno disposto una coppia di studenti per ciascuna delle tre fasi descritte e preparato i propri interventi. Prima dell'inaugurazione della mostra la visita guidata è stata testata attraverso simulazioni in presenza del tutor interno che ha corretto e migliorato contenuti e fluidità dell'esposizione. La richiesta agli studenti di svolgere parte del lavoro in autonomia, opportunità rara se non

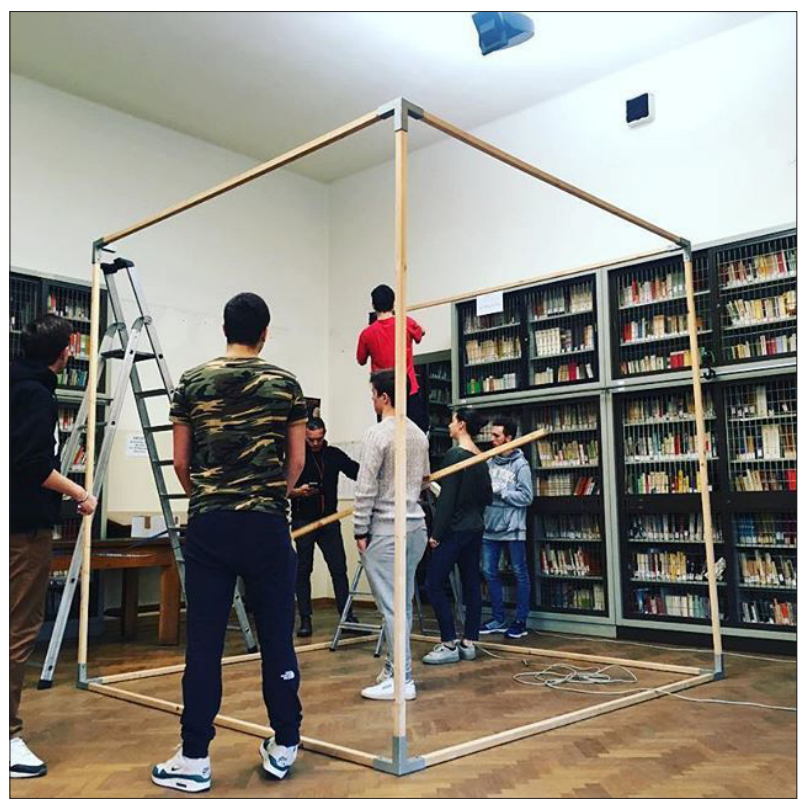

Lavori di montaggio in biblioteca.

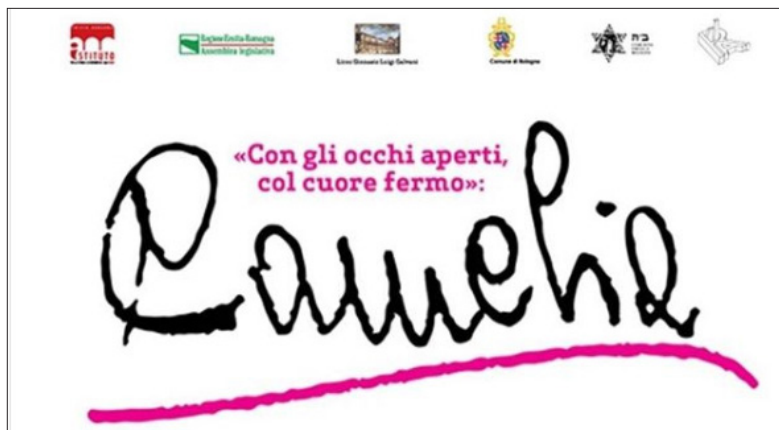

\section{FEBBRAIO - 20 MAGGIO}

Mostra dedicata a Camelia Matatia, una ragazza ebrea che ha vissuto nel periodo delle leggi razziali.

PRESSO LA BIBLIOTECA DEL LICEO A.RIGHI Viale Pepoli 3, Bologna II lavoro è stato realizzato da alunni del Liceo Galvani
Presentano la mostra gli alunni della 5B del Liceo A.Righi

Per info e prenotazioni:

(3) Following Camelia's life

(O) info_mostra_camelia

$M$ info.mostracamelia@gmail.com

Il volantino della mostra. 
completamente assente nell'usuale routine scolastica, ha portato, come sarà trattato nella sezione finale, risultati diversi.

\section{Le visite guidate}

Il 29 gennaio 2018 la VB ha inaugurato presso il Liceo Righi Following Camelia's life. Il pubblico della prima visita era formato da membri dell'Assemblea Emilia-Romagna, alcune classi dell'istituto e, per la prima volta, dai coniugi Matatia che non avevano ancora potuto fruire del risultato finale del progetto del Galvani.

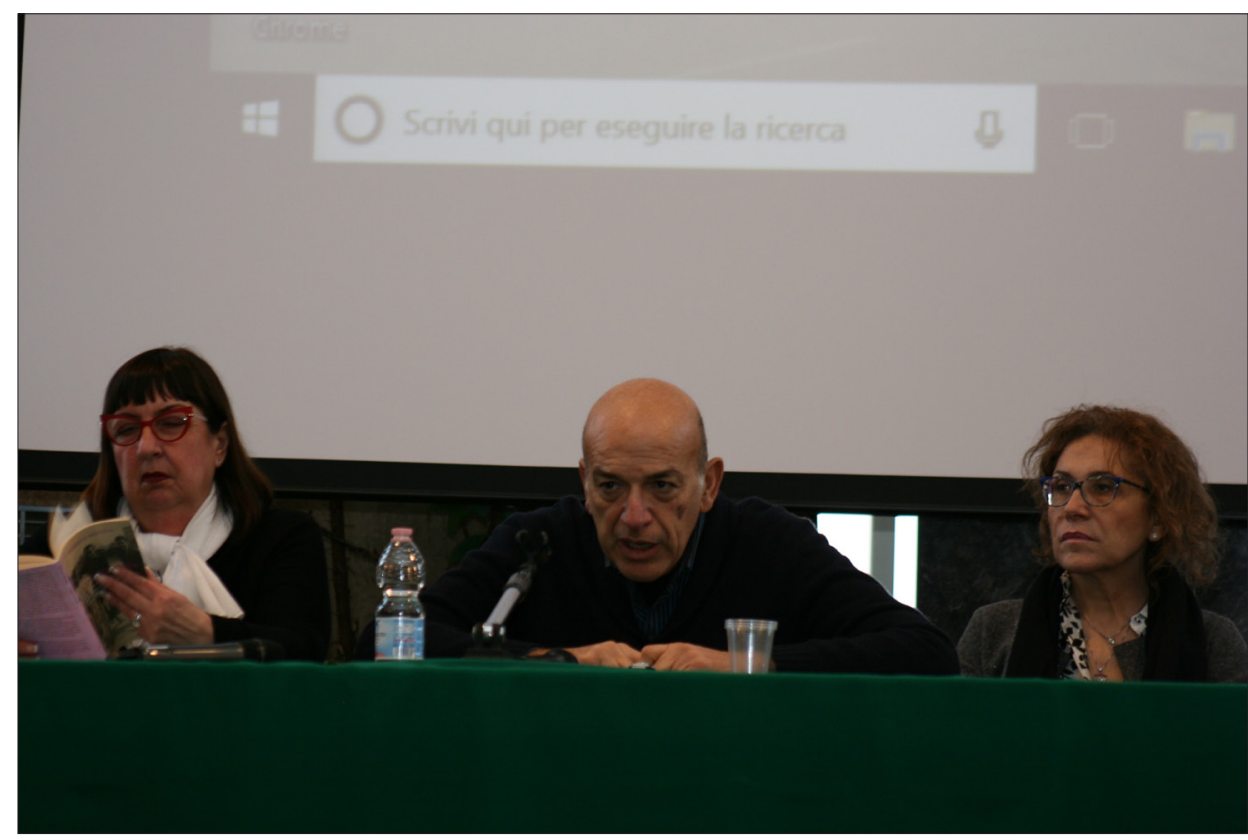

Roberto Matatia nell'Aula Magna del Liceo Righi.

L'inaugurazione, preceduta da un incontro di confronto tra i coniugi Matatia e gli studenti del liceo riguardante la storia di Camelia e il tema delle leggi razziali, ha avuto pieno successo: nella mattinata culmine del loro progetto, gli studenti hanno mostrato i risultati del loro percorso di alternanza scuola-lavoro e riscosso un feedback positivo da parte del diversificato pubblico presente, che ha lasciato per iscritto i propri sentiti e non banali commenti.

Nei mesi successivi, in date selezionate tra il 12 febbraio e il 20 maggio, la classe ha proseguito autonomamente la gestione della mostra, inviando periodicamente al tutor esterno il resoconto del lavoro svolto. 


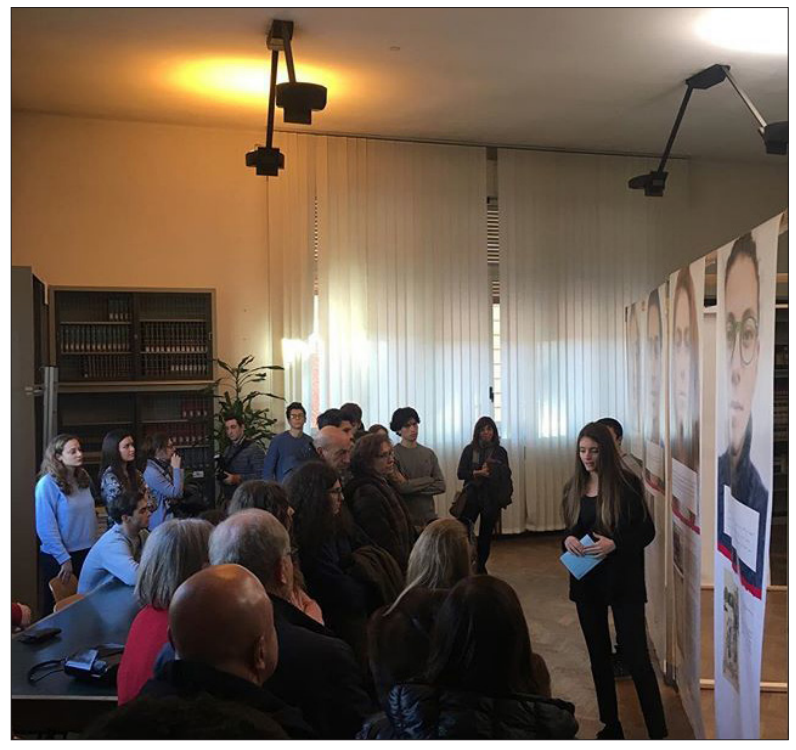

L'inaugurazione della mostra.
Il calendario delle esposizioni, in accordo con le disponibilità degli studenti guide, ha coinvolto la settimana dei recuperi del liceo, le mattinate delle assemblee d'istituto e i pomeriggi dei colloqui di ricevimento dei genitori, per un totale di circa una quindicina di visite. Gli studenti hanno talvolta arricchito le visite leggendo alcune pagine de I vicini scomodi per favorire il coinvolgimento dei visitatori.

Premesso che le dimensioni del pubblico dell'inaugurazione non fossero plausibilmente ripetibili, le esposizioni successive hanno comunque goduto di una soddisfacente partecipazione, diversa a seconda delle circostanze in cui erano organizzate e i visitatori coinvolti. Se la narrazione della vita di Camelia è stata ge-

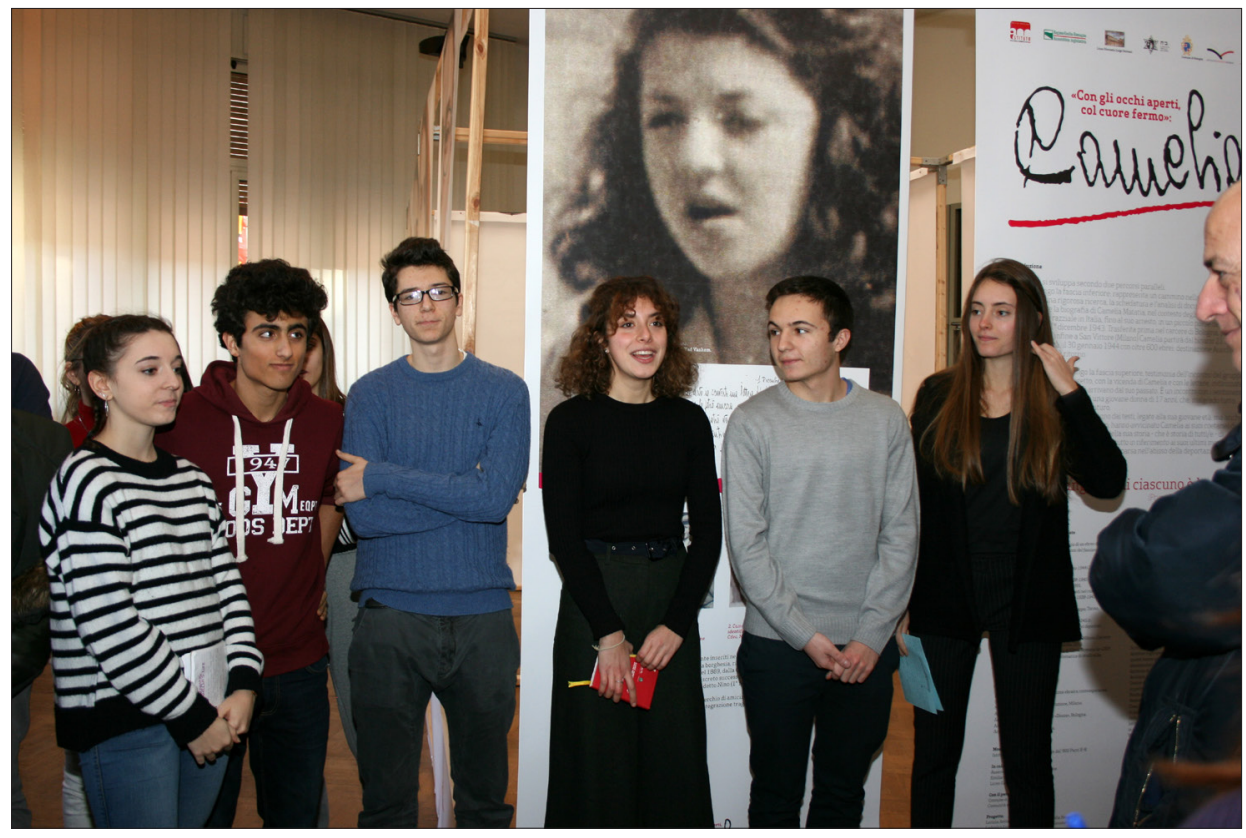

Gli studenti-guide 
neralmente apprezzata da genitori e classi accompagnate da professori del Righi (in un caso anche delle scuole medie Guinizelli), le visite guidate si sono rivelate più problematiche nel corso delle assemblee d'istituto. Questo tipo di giornate, pensate per promuovere una didattica alternativa attraverso attività proposte $\mathrm{e}$ gestite dagli studenti su temi a scelta, rappresentano in apparenza l'occasione perfetta per lo svolgimento delle visite guidate di Following Camelia's life. Ciò nonostante, come sanno bene gli studenti, nel concreto le assemblee sono spesso giornate vuote, a cui molti partecipano solo per non far registrare un'assenza, ma senza aderire alle, solitamente poche, attività proposte. In questo tipo di contesto la biblioteca scolastica, sede della mostra, diventa il luogo dove studiare, senza prender parte alle attività. Consapevoli di questa dinamica, le guide hanno trovato con i compagni un compromesso, secondo cui hanno presentato ai soli pochi interessati la mostra e lasciato gli altri liberi di occupare parte della biblioteca a patto di restare in silenzio. In ogni caso, il ridotto pubblico effettivo delle assemblee, che ha compreso anche un gruppo di studenti universitari ex righini, ha apprezzato il lavoro svolto dalla VB.

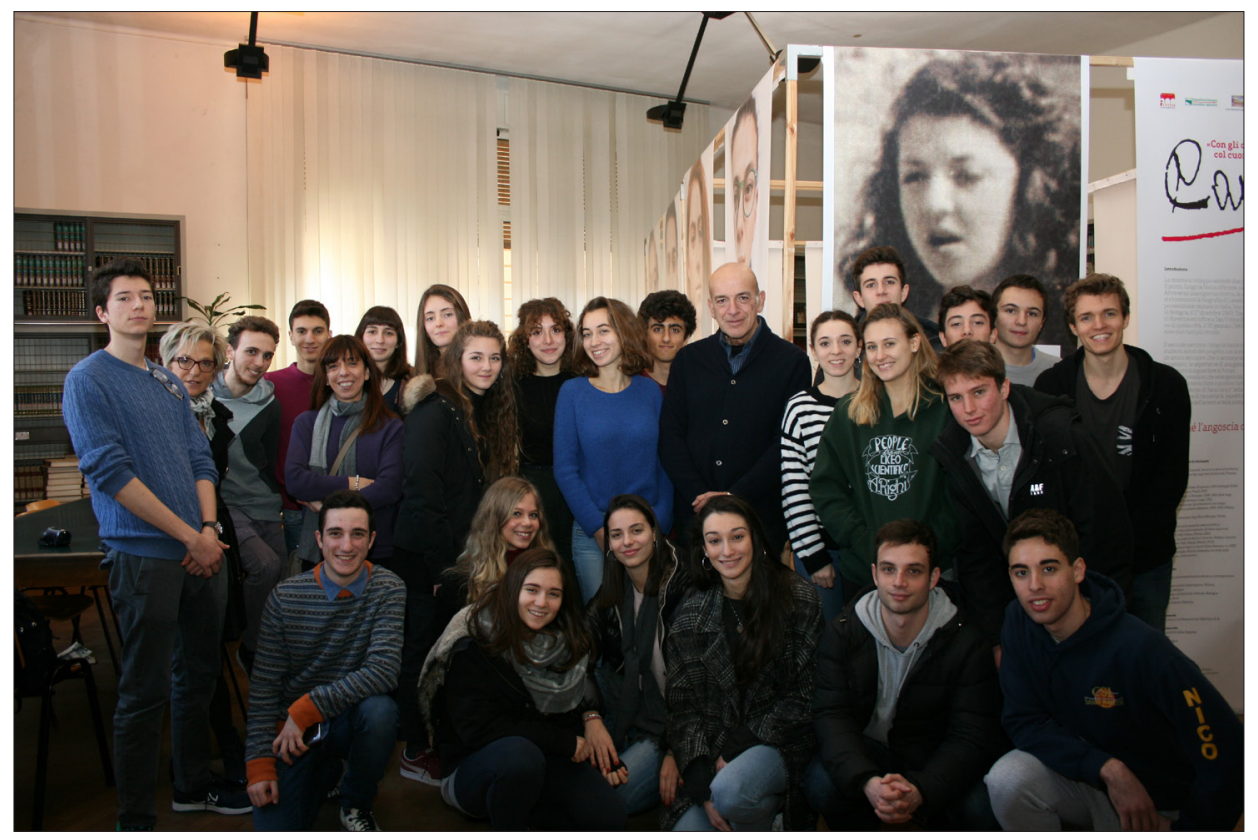

La VB insieme ai tutor e Roberto Matatia. 


\section{Risultati e riflessioni}

Il percorso di alternanza scuola-lavoro incentrato su Following Camelia's life è stato, a giudizio di professori e studenti, entrambi pregiudizialmente scettici verso l'alternanza scuola-lavoro, globalmente positivo. Tra i docenti, sia il tutor interno che i colleghi spettatori sono stati colpiti dalle risposte prodotte dagli studenti alle richieste di creatività e organizzazione necessarie all'attività, dalla capacità di ripensamento e trasformazione dell'ambiente della biblioteca scolastica alle appassionate e professionali esposizioni delle guide. Anche per quanto riguarda il pubblico di studenti e genitori, rifacendosi ai numerosi commenti lasciati a fine visita, l'obiettivo di sensibilizzazione storica e avvicinamento empatico è da considerare conseguito. Va purtroppo menzionata anche la presenza di alcuni post-it anonimi di contenuto volgare e spregiativo che sono stati considerati inopportuni e rimossi.

Per contro, il progetto di Following Camelia's life non ha convinto l'intero gruppo classe, diviso, come sempre, tra soddisfazione e indifferenza rispetto all'attività. Parte degli attriti si è risolto nella tipica resistenza degli studenti verso l'alternanza scuola-lavoro, contestata dalla nascita con l'accusa di sottrarre ore alla tradizionale didattica per attività e di non sempre certa utilità. Se, in effetti, il secondo semestre della quinta richiede più tempo da dedicare allo studio, entrambi i mediamente buoni esiti conseguiti dal progetto e dall'esame di stato dimostrano come le due attività siano state di fatto conciliabili. In ogni caso, per evitare questa facile polemica, il percorso potrebbe essere riproposto alle classi quarte, oppure anticipato al primo trimestre della quinta. Per quanto riguarda le critiche più inerenti al progetto specifico, si evidenzia un solo vero punto di debolezza: il mancato coinvolgimento nell' arco dello svolgimento del lavoro dell'intero gruppo classe. Mentre nella fase iniziale di preparazione il lavoro è stato condiviso, dopo la prima mostra l'attività è stata portata avanti praticamente dalle sole guide. Le 30 ore di alternanza conteggiate per ogni studente non hanno rappresentato un riscontro effettivo del lavoro di ciascun studente, che, a seconda dei casi, ne ha svolto una quantità sensibilmente maggiore o minore.

Questo si deve in parte alla scarsa volontà di coinvolgimento di alcuni ragazzi e in parte all'impostazione del percorso, la cui fondamentale e pregevole premessa di spingere all'autonomia nella gestione del lavoro comporta per sua natura risultati altalenanti: alcuni rispondono allo stimolo e raccolgono la sfida con impegno, dimostrando di saper dare contributi originali come il video promozionale o l'aggiunta della raccolta dei commenti del pubblico alla mostra; altri colgono l'occasione per fare meno sforzo possibile, esaurendo il loro incarico nei mini- 
mi termini richiesti. Sebbene questo esito sia in gran parte inevitabile - sarebbe ingenuo attendersi da un'intera classe una totale ed entusiasta partecipazione ad un'attività didattica, soprattutto se di alternanza scuola-lavoro - è tuttavia possibile tentare di migliorare la qualità dello stimolo proposto agli studenti per invogliarne un maggior numero alla partecipazione attiva.

Nel caso di Following Camelia's life, il gruppo delle guide è stato quello sottoposto alla sfida più impegnativa e che, forse proprio in virtù di questo, ha lavorato meglio. La maggior serietà con cui è stato svolto l'incarico non è spiegabile soltanto nel merito degli interpreti, ma anche nel maggior fascino del compito loro assegnato. Per quanto la questione sia soggettiva, è più facile che uno studentemedio trovi maggiore interesse nell'essere protagonista di una presentazione che non nel gestire prenotazioni via email. Per una più equa distribuzione del lavoro e un più ampio coinvolgimento della classe, potrebbe dunque rivelarsi proficuo provare a dividere l'incarico di guide delle visite tra tutti gli studenti. Per esempio, dopo la fase condivisa di preparazione della mostra, i gruppi della classe (4-6 alunni circa) potrebbero organizzare un calendario delle esposizioni in modo che ciascuno sia responsabile di una quantità minima di visite guidate nell'arco di una determinato periodo di tempo. Questa strategia garantirebbe lo svolgimento di un maggior numero di visite guidate della mostra rispetto a quelle organizzabili da un solo gruppo.

Al di là di questo utile accorgimento, il percorso didattico di Following Camelia's life rappresenta una stimolante attività meritevole di future riproposizioni: è un'originale interpretazione della discussa alternanza scuola-lavoro che permette di portare dentro e fuori dalle scuole il delicato tema storico delle leggi razziali nella sua dimensione memoriale, rendendo gli studenti costruttori attivi di cultura.

\section{Risorse on line}

Video promozionale della mostra

https://drive.google.com/file/d/1z3uCWaOYt1K6J_N9giD_k8nzRuF3AuFR/view

Pagina Facebook

https://www.facebook.com/Following-Camelias-life-1297021180403456/

Account Instagram

https://www.instagram.com/info_mostra_camelia/ 